\title{
Thanks to all those who reviewed for Journal of Medical Case Reports in 2014
}

Michael R Kidd

\section{Contributing reviewers}

A peer-reviewed journal would not survive without the generous time and insightful comments of the reviewers, whose efforts often go unrecognized. Although final decisions are always editorial, they are greatly facilitated by the deeper technical knowledge, scientific insights, understanding of social consequences, and passion that reviewers bring to our deliberations. For these reasons, the Editor-in-Chief and staff of the journal warmly thank the 906 reviewers whose comments helped to shape Journal of Medical Case Reports, for their invaluable assistance with review of manuscripts for the journal in Volume 8 (2014).

Souheil Abdel Nour

USA

\section{Ahmad Abdelkarim}

USA

Rohana Abdul Ghani

Malaysia

Koji Abe

Japan

Arnis Abolins

Latvia

Edward Abraham

USA

Yushi Adachi

Japan

Kensuke Adachi

Japan

Roberto Adani

Italy

Omotayo Adesiyun

Nigeria

Christian Agard

France
Elugwuraonu Agbakwuru

Nigeria

Varun Agrawal

USA

Tasnim Ahsan

Pakistan

Toshimi Aizawa

Japan

Sinem Akgül

Turkey

Esen Akpek

USA

Gabriel Alcala

Colombia

Mohammad Al-Haggar

Egypt

Waleed Alkhudair

Saudi Arabia

Fawzia Al-Otaibi

Saudi Arabia

Murat Alper

Turkey
Mohannad Alqudah

Jordan

Fatih Altunrende

Turkey

Hernando Raphael Alvis Miranda Colombia

Theodore Anagnostou

Greece

Olympia Anastasiou

Germany

Uzel Andre-Pierre

Guadeloupe

Manjula Annappa

UK

Alexandros Apostolopoulos

Greece

Slobodan Apostolski

Serbia

Michele Arcopinto

Italy

Sandro Ardizzone

Italy

Correspondence: michael.kidd@flinders.edu.au

Faculty of Health Sciences, Flinders University, GPO Box 2100, Adelaide, SA

5001, Australia 
Guillaume Armengol

France

Eleni Arnaoutoglou

Greece

Vaithi Arumugaswami

USA

Keyhan Ashrafi

Iran

Irene Asouhidou

Greece

Jaromir Astl

Czech Republic

Andrew Atkinson

USA

Stephen Attard

Malta

Brian Austin

UK

Mehmet Aydogan

Turkey

Hüseyin Ayhan

Turkey

Yavuz Ayhan

Turkey

Ibrahim Azboy

Turkey

Hideki Azuma

Japan

Hisatoshi Baba

Japan

Yuh Baba

Japan

Hector Baillie

Canada

Salar Bakhtiyari

Iran

Khaled Balam

Egypt

Alberto Baldini

Italy

Nikolaos Baltayiannis

Greece

Upali Banagala

Sri Lanka
Paul Anthony Banaszkiewicz

UK

Paulina J.M. Bank

Netherlands

Giorgio Barbarini

Italy

Mary Barry

Ireland

Sandip Basu

India

Mihaela Batke

USA

Mark Bechtel

USA

George Beiko

Canada

Diana Bell

USA

Reuben Benjamin

UK

Ronald Benveniste

USA

Alan Berger

Canada

Thomas Berger

Switzerland

Robert Bergholz

Germany

Achim Berthele

Germany

Rajesh Bhagat

USA

Vish Bhattacharya

UK

Shahaab Bhatti

UK

Deepak Bhonagiri

Australia

Can Bicmen

Turkey

Jovan Bila

Serbia

Ashish Bindra

India
Jean Bismuth

USA

Benoit Blondeau

USA

Stijn Blot

Belgium

Paolo Boffano

Italy

Massimo Bonacchi

Italy

Hamouda Boussen

Tunisia

David Boyer

USA

Colin Bradley

Ireland

Patrick Bradley

UK

Elizabeth Brant

USA

Milan Brazdil

Czech Republic

Massimo Breccia

Italy

Olivier Brehant

France

Raoul Breitkreutz

Germany

Vesna Breznik

Slovenia

Giuseppe Brisinda

Italy

Konrad Brockmeier

Germany

Claudia Bruè

Italy

Manjunatha Bs

India

Alicja Buczek

Poland

Soetjiningsih Budiarsa

Indonesia

Ertan Bulbuloglu

Turkey 
Orhan Bulut

Denmark

Jean Burr

USA

Cen Bytyqi

Albania

Maurizio Calcagni

Switzerland

Massimo Caldarelli

Italy

Mario Camarda

Italy

Danny Camfferman

Australia

Giovanni Cammarota

Italy

Fabio Cesare Campanile

Italy

Alessandro Cannavale

Italy

Steve Carlan

USA

Pilar Carranza

Mexico

Santos Castañeda

Spain

Joaquin Castro

Spain

Juan Cataño

Colombia

Niyazi Cebi

Germany

Francesco Ceccarelli

Italy

Marco Cei

Italy

Giuliano Cerulli

Italy

Camila Cespedes

Colombia

Parin Chaivisuthangkura

Thailand

Shanmuganathan Chandrakasan

USA
Shi-Min Chang

China

Philippe Chanson

France

Rene Chapot

Germany

Alexandros Charalabopoulos

UK

Keerati Chareancholvanich

Thailand

Stylianos Chatzipanagiotou

Greece

Nikolaos Chavouzis

Greece

Baoan Chen

China

Ying-Sheue Chen

Taiwan

Domenico Chirchiglia

Italy

Joanne Chiu

Hong Kong

Kyu-Sup Cho

South Korea

Anand Chockalingam

USA

Evangelos Cholongitas

Greece

Tumul Chowdhury

Canada

Sara Ciccone

Italy

Mislav Cimic

Croatia

Kristl Claeys

Germany

Christelle Clement-Duchene

France

Joannes Clerinx

Belgium

David Cloke

UK

Paul Cockwell

UK
Stephan Cohen-Bacrie

France

Demosthenes Cokkinos

Greece

Andrés Combalia

Spain

Stefano Congiu

UK

Patricia Conville

USA

Ruggero Corso

Italy

Demet Coskun

Turkey

Ana Costa

Brazil

Anna Crocco

Italy

Adam Croft

UK

Kimberly Crowder

USA

Salvador Cruz-Flores

USA

Francisco Cuellar-Ambrosi

Colombia

Vatsla Dadhwal

India

Antonio D'alessandro

Italy

Maurizio D'amario

Italy

Abdul Qayoom Dar

India

Giuseppe Dattilo

Italy

Carlo De Angelis

Canada

Emmanuel De Laere

Belgium

Francesco Giuseppe De Rosa Italy

Dulantha De Silva

Sri Lanka 
Juan Carlos De Vicente

Spain

Masataka Deie

UK

Andreas K. Demetriades

UK

Ismail Demiryilmaz

Turkey

Francesco Dentali

Italy

Aparna Deshpande

India

Giovambattista Desideri

Italy

Mark Detweiler

USA

Amar Dhand

USA

Luca Di Antonio

Italy

Andrea Di Bernardo

Italy

Hendrik Dienemann

Germany

Daan Dierickx

Belgium

Panagiotis Dikeakos

Greece

Ludovico Docimo

Italy

Stefanos Dokas

Greece

Bessy Domi

Greece

Frank Domino

USA

William Donaldson

UK

Kieran Doran

Ireland

Dimitrios Dougenis

Greece

Panagiotis Drakopoulos

Switzerland
Georgios Drosos

Greece

Charalambos Drossos

Greece

Balazs Duga

Hungary

Gregory Dumanian

USA

Reinhard Dummer

Switzerland

Martin Dunser

Austria

Nizam Duran

Turkey

Onur Burak Dursun

Turkey

Amaka Edeani

USA

Suzanne Edmunds

USA

Dr Meera Ekka

India

Fatih Eksioglu

Turkey

Ghada El Sagheer

Saudi Arabia

Aravindan Elangovan

India

Abdelhamid Elgazzar

Kuwait

Adel El-Naggar

USA

Mohamed Emad

Egypt

Tze Kiong Er

Taiwan

Silvano Esposito

Italy

Giuseppe Maria Ettorre

Italy

Mohamed Fahmy

Egypt

Lee Fairlie

South Africa
Rafik Fanous

UK

Silvia Fargion

Italy

Eriberto Farinella

Italy

James Farmer

Canada

Magi Farre

Spain

Shoaib Faruqi

UK

Hasan Fattah

USA

Chris Fearne

Malta

Pasquale Ferrante

Jamaica

Mara Ferrara

Italy

Mauricio Ferri

Canada

Nicholas Fischer

New Zealand

Horace Fletcher

Jamaica

Panagiota Flevari

Greece

Andrés Fonnegra

Colombia

Denis Leonardo Fontes Jardim Brazil

Alessia Fornoni

USA

Elias Fotiadis

Greece

Christina Fotopoulou

UK

Claudio Fozza

Italy

Gabriele Fragasso

Italy

Dario Frank

Germany 
Florian Friedmacher

Ireland

Johan H.M. Frijns

Netherlands

\section{Lorenzo Fuccio}

Italy

Masashi Fukushima

Japan

Ioannis Galanis

Greece

Paolo Gallipoli

UK

Sean Galvin

Australia

T. Clark Gamblin

USA

Prasanth Ganesan

India

Musa Abubakar Garbati

Saudi Arabia

Rakesh Garg

India

Enrique Garrido

UK

Bogdan Gaspar

Romania

Amy Gassama Sow

Senegal

Danielle Gatti

USA

Elzbieta Gawrych

Poland

Roman Gebauer

Germany

Ralph George

Canada

Petros Georgopoulos

Greece

Mehdi Ghasemi

USA

Mohammad Ghasemi-Rad

Iran

Christopher Ghazala

UK
Jamileh Ghoddusi

Iran

Kemel Ahmed Ghotme Ghotme

Colombia

Stilianos Giannakopoulos

Greece

Paul Gibson

Canada

Don Gilden

USA

Ritu Gill

USA

Matteo Giorgi

Italy

Olga Giouleme

Greece

Stefano Giulieri

Switzerland

Irina Giurgea

France

Panagiotis Givissis

Greece

Markus Gödel

Germany

Bee See Goh

Malaysia

Cem Gökçen

Turkey

Elizabeth Golesic

Canada

Rodrigo Gomes Da Silva

Brazil

John Gonder

Canada

Laura González-García

Spain

Ponnampalam Gopalakrishnakone

Singapore

David Gorard

UK

Martijn Pieter Gosselink

Netherlands

Pierre Goussard

South Africa
Tarang Goyal

India

Elvira Grandone

Italy

Davide Grassi

Italy

Victor Grech

Malta

Neil Greening

UK

Sandeep Grover

USA

Beatriz Guglieri

Spain

Farshid Guilak

USA

Dorothy Gujral

UK

Niti Gulati

India

Selma Guler

Turkey

Anju Gupta

India

Gaurav Gupta

USA

Manish Gupta

India

Swati Gupta Bhattacharya

India

Kathleen Gura

USA

José-María Gutiérrez

Costa Rica

Melih Güven

Turkey

Vijay Hadda

India

Hind Hadi

Iraq

Sanghoon Han

South Korea

Tharangrut Hanprasertpong

Thailand 


\author{
Rehan Haq \\ India \\ K.V.S. Hari Kumar
India \\ Uma Hariharan \\ India \\ Claire Harrison \\ UK \\ Yamada Harumoto \\ Japan \\ Junichi Hashimoto \\ Japan
}

Miroslav Haspl
Croatia

Iffat Hassan

India

Michael Hawkes

Canada

Reda Hemida

Egypt

Patrick Henn

Ireland

Ruth Heying

Belgium

Catherine Hill

Australia

Andreas Himmelmann

Switzerland

Simon Hjerrild

Denmark

Martin Hoenigl

Austria

Iva Hojak

Croatia

Sung-Tae Hong

South Korea

Jae Young Hong

South Korea

Hironobu Hoshino

Japan

Sion Houri

Israel

Sun Huh

South Korea
Robert Hunter

UK

Mark Huntington

USA

Richard Huntsman

Canada

Nexhmi Hyseni

Kosovo

Giorgio Iannetti

Italy

Kenneth Iczkowski

USA

Abdullah Inal

Turkey

Philip Ind

UK

Yasushi Innami

Japan

Sebastian Ionescu

Romania

Fumihiro Ishida

Japan

Jafar Jafari

UK

Amita Jain

India

Neetu Jain

India

Praveen Jajoria

USA

Jens Jakob

Germany

Prashant Jani

Canada

Anis Jellad

Tunisia

Seong Woo Jeon

South Korea

Woo-Jin Jeong

South Korea

Verónica Jeraldo

Brazil

Andrei Joaquim

Brazil
Ken Johkura

Japan

Brian Joondeph

USA

Tatjana Josifova

Switzerland

Eui Dal Jung

South Korea

Fevziye Kabukcuoglu

Turkey

Anish P. Kadakia

UK

Abdullah Kaki

Saudi Arabia

Arjun Kakrani

India

Evagelos Kalfarentzos

Greece

Michal Kaliszan

Poland

Takeharu Kanazawa

Japan

Gokul Vignesh Kanda Swamy

UK

Hideaki Kanemura

Japan

Yoshihiko Kanno

Japan

Amrinderjit Kanwar

India

Rene Kanza

Canada

Emmanouil Ioannis Kapetanakis

Greece

Pinar Karabagli

USA

Nesrin Karabul

Germany

Panagiotis Karanis

Germany

Mehdi Karkouri

Morocco

Maria Karouki

Greece 
Suneth Karunarathne

Sri Lanka

Imad Kassis

Israel

Masaya Kawai

Japan

Leticia Kawano-Dourado

Brazil

Cemil Kayali

Turkey

Carolin Kayser

Germany

Donald Kearns

USA

Jean Keddissi

USA

Pierre Kehr

France

Martina Kelly

Canada

Michael Kelly

USA

Ozgur Kemik

Turkey

Melita Kenealy

Australia

Mahmoud Khattab

Egypt

S. Guan Khoo

Ireland

Teruyo Kida

Japan

Katarzyna Kilis-Pstrusinska

Poland

Tae-Heung Kim

South Korea

Hee Jun Kim

South Korea

Won-Jang Kim

South Korea

Seong Heon Kim

South Korea

Ryo Kimura

Japan
Ana Cristina King Martinez

Mexico

Takeshi Kinjo

Japan

Laszlo Kiraly

United Arab Emirates

Boris Kirshtein

Israel

Kamal Kishore

USA

Hiroaki Kitagawa

Japan

Masaru Kitamura

Japan

Ingo Kleiter

Germany

Richard Knight

UK

Christian Koch

USA

Puneet Kochhar

India

Diamantis Kofteridis

Greece

Anna Kohn

Italy

Mehmet Hanifi Kokaçya

Turkey

Matthias König

Switzerland

Satoshi Kono

Japan

Marina Kontogiorgi

Greece

Marcela Kopacova

Czech Republic

Ioannis Kornezos

Greece

Chris Kosmidis

UK

Prakash Kotagal

USA

Anastasia Kotanidou

Greece
Efstathios Kotidis

Greece

Kalliopi Kotsa

Greece

Katerina Kotzampassi

Greece

Georgios Kouliatsis

Greece

Gregory Kouraklis

Greece

Ioannis Koutelidakis

Greece

Euphrosyni Koutsouraki

Greece

Anastasios Koutsovasilis

Greece

Gopal Kowdley

USA

Roland Kreis

Switzerland

Uma Krishnamoorthy

UK

David Krpata

USA

Ikuko Kubokawa

Japan

Senanayake Kularatna Sri Lanka

Ajay Kumar

India

Mahesh Kumar

UK

Niranjan Kumar

Kuwait

Shivaani Kummar

USA

Motoki Kuramochi

Japan

Mertihan Kurdoglu

Turkey

Vivek Kute

India

Yok-Lam Kwong

Hong Kong 
Severin Laeuchli

Switzerland

De-Hua Lai

China

Ka On Lam

Hong Kong

William Laskin

USA

Ernest Lau

UK

Andreas Lazaris

Greece

Laurianne Le Gloan

France

David Lebeaux

France

Jaekwang Lee

South Korea

Claudio Letizia

Italy

Yuen-Fai Leung

Hong Kong

Roland Leung

Hong Kong

Liran Levin

Israel

Miles Levy

UK

Chengyao Li

China

Wei Li

China

Xiaoling Liang

China

Ying Liang

China

Christos Liapis

Greece

Chester Lie

Hong Kong

Sue Lightman

UK

Lassi Liikkanen

Finland
Jin-Ching Lin

Taiwan

James Lin

USA

Tung-Liang Lin

Taiwan

Gopal Lingam

Singapore

Katerina Linhartova

Czech Republic

Qun Liu

China

Bingqian Liu

China

Tianrun Liu

China

Eddie Liu

Canada

Quan Liu

China

Tsafrir Loebl

Israel

Jonathan Loughead

UK

Chih-Ming Lu

Taiwan

Shue-Fen Luo

Taiwan

Stefan Lüth

Germany

Ângela Cristina Malheiros Luzo

Brazil

Hatsuhiko Maeda

Japan

Yoshito Maeno

Japan

John Maesaka

USA

Rama Maganti

USA

Magboul Magboul

USA

Bridget Maher

Ireland
Joseph Maher

USA

Kohei Makita

UK

Demosthenes Makris

France

Nilesh Makwana

UK

Vijay Malpathak

India

Efstratios Maltezos

Greece

Vincenzo Dario Mandato

Italy

Charles Mango

USA

Nicholas Mann

UK

Surendra Mantoo

Singapore

Emilia Manzato

Italy

Giorgos Margaritopoulos

Greece

Athanasios Marinis

Greece

Gian Luigi Marseglia

Italy

Ian Marshall

Australia

Thomas Martin

UK

Hirofumi Maruyama

Japan

Helena Marzo-Ortega

UK

Lorenzo Masi

Italy

Ibrahim Masoodi

Saudi Arabia

Sotiria Mastoraki

Greece

Nilesh Mathuria

USA 


\begin{tabular}{|c|c|c|}
\hline Ratko Matijevic & Giancarlo Micheletto & Benvon Moran \\
\hline Croatia & Italy & Canada \\
\hline $\begin{array}{l}\text { Andreas Mavrogenis } \\
\text { Greece }\end{array}$ & $\begin{array}{l}\text { Atsuya Miki } \\
\text { Japan }\end{array}$ & $\begin{array}{l}\text { Carthage Moran } \\
\text { Ireland }\end{array}$ \\
\hline $\begin{array}{l}\text { Michinori Mayama } \\
\text { Japan }\end{array}$ & $\begin{array}{l}\text { Ioannis Mikoniatis } \\
\text { Greece }\end{array}$ & $\begin{array}{l}\text { Mohib Morcos } \\
\text { Canada }\end{array}$ \\
\hline $\begin{array}{l}\text { Joni Mazza-Mccrann } \\
\text { USA }\end{array}$ & $\begin{array}{l}\text { Stephanos Milias } \\
\text { Greece }\end{array}$ & $\begin{array}{l}\text { Tom Moreels } \\
\text { Belgium }\end{array}$ \\
\hline $\begin{array}{l}\text { Maria Antonietta Mazzei } \\
\text { Italy }\end{array}$ & $\begin{array}{l}\text { Michael Mimouni } \\
\text { Israel }\end{array}$ & $\begin{array}{l}\text { Umberto Morelli } \\
\text { France }\end{array}$ \\
\hline $\begin{array}{l}\text { Valeria Mazzi } \\
\text { Italy }\end{array}$ & $\begin{array}{l}\text { Akihito Minamide } \\
\text { Japan }\end{array}$ & $\begin{array}{l}\text { Arsim Morina } \\
\text { Kosovo }\end{array}$ \\
\hline $\begin{array}{l}\text { Sam Mbulaiteye } \\
\text { USA }\end{array}$ & $\begin{array}{l}\text { Roberta Minelli } \\
\text { Italy }\end{array}$ & $\begin{array}{l}\text { Hideo Morioka } \\
\text { Japan }\end{array}$ \\
\hline $\begin{array}{l}\text { Meer Chisthi Meeran Pillai } \\
\text { India }\end{array}$ & $\begin{array}{l}\text { Alberto Minetti } \\
\text { Italy }\end{array}$ & $\begin{array}{l}\text { Silvia Morlino } \\
\text { Italy }\end{array}$ \\
\hline $\begin{array}{l}\text { Bruno Megarbane } \\
\text { France }\end{array}$ & $\begin{array}{l}\text { Michele Miraglia Del Giudice } \\
\text { Italy }\end{array}$ & $\begin{array}{l}\text { Eleni Mouloudi } \\
\text { Greece }\end{array}$ \\
\hline $\begin{array}{l}\text { Enawgaw Mehari } \\
\text { USA }\end{array}$ & $\begin{array}{l}\text { George Mistriotis } \\
\text { Greece }\end{array}$ & $\begin{array}{l}\text { Ramanuj Mukherjee } \\
\text { India }\end{array}$ \\
\hline $\begin{array}{l}\text { Changlin Mei } \\
\text { China }\end{array}$ & $\begin{array}{l}\text { Jayanta Mitra } \\
\text { India }\end{array}$ & $\begin{array}{l}\text { Nicola Mumoli } \\
\text { Italy }\end{array}$ \\
\hline $\begin{array}{l}\text { Jurka Meichtry } \\
\text { Switzerland }\end{array}$ & $\begin{array}{l}\text { Amit Mittal } \\
\text { India }\end{array}$ & $\begin{array}{l}\text { James Munthali } \\
\text { Zambia }\end{array}$ \\
\hline $\begin{array}{l}\text { Shimon Meretyk } \\
\text { Israel }\end{array}$ & $\begin{array}{l}\text { Seiki Miura } \\
\text { Japan }\end{array}$ & $\begin{array}{l}\text { Giuseppe Murdaca } \\
\text { Italy }\end{array}$ \\
\hline $\begin{array}{l}\text { Andrew Merkur } \\
\text { USA }\end{array}$ & $\begin{array}{l}\text { Hiroshi Miyamoto } \\
\text { USA }\end{array}$ & $\begin{array}{l}\text { Thibaut Murez } \\
\text { France }\end{array}$ \\
\hline $\begin{array}{l}\text { Linda Metaxa } \\
\text { Greece }\end{array}$ & $\begin{array}{l}\text { Muhajir Mohamed } \\
\text { Australia }\end{array}$ & $\begin{array}{l}\text { Heather Murphy-Lavoie } \\
\text { USA }\end{array}$ \\
\hline $\begin{array}{l}\text { Salvatore Micali } \\
\text { Italy }\end{array}$ & $\begin{array}{l}\text { Mohamed Ali Ahmed Mohamed } \\
\text { Egypt }\end{array}$ & $\begin{array}{l}\text { Nagarajan Muthialu } \\
\text { UK }\end{array}$ \\
\hline $\begin{array}{l}\text { Othon Michail } \\
\text { Greece }\end{array}$ & $\begin{array}{l}\text { Rhajaoui Mohamed } \\
\text { Morocco }\end{array}$ & $\begin{array}{l}\text { Seema Nagpal } \\
\text { USA }\end{array}$ \\
\hline $\begin{array}{l}\text { Jaroslav Michalek } \\
\text { Czech Republic }\end{array}$ & $\begin{array}{l}\text { Khalid Mohammad } \\
\text { USA }\end{array}$ & $\begin{array}{l}\text { Akitoshi Nakashima } \\
\text { Japan }\end{array}$ \\
\hline $\begin{array}{l}\text { Pavel Michalek } \\
\text { Czech Republic }\end{array}$ & $\begin{array}{l}\text { Ali Mokhtarpour } \\
\text { Malaysia }\end{array}$ & $\begin{array}{l}\text { Raj Nandi } \\
\text { UK }\end{array}$ \\
\hline $\begin{array}{l}\text { Pavel Michalek } \\
\text { UK }\end{array}$ & $\begin{array}{l}\text { Felix Momm } \\
\text { Germany }\end{array}$ & $\begin{array}{l}\text { Sameer Naranje } \\
\text { USA }\end{array}$ \\
\hline $\begin{array}{l}\text { Antonios Michalopoulos } \\
\text { Greece }\end{array}$ & $\begin{array}{l}\text { Suneeta Monga } \\
\text { Canada }\end{array}$ & $\begin{array}{l}\text { Kiran Narreddy } \\
\text { USA }\end{array}$ \\
\hline $\begin{array}{l}\text { Nickolaos Michalopoulos } \\
\text { Greece }\end{array}$ & $\begin{array}{l}\text { Guillermo Monsalve } \\
\text { Colombia }\end{array}$ & $\begin{array}{l}\text { Asma Nasim } \\
\text { Pakistan }\end{array}$ \\
\hline
\end{tabular}


Mc Nassogne

Belgium

Sundaram Natarajan

India

Ramon Navarro

United Arab Emirates

Andrew Neviaser

USA

Jarungchit Ngamphaiboon

Thailand

Korahanis Nicolas

France

Lluís Nisa

Switzerland

Aamer Nisar

UK

Tetsuro Nishimura

Japan

Pavel Nockel

USA

Ahmad Nofal

Egypt

Hilali Noordeen

UK

Aqodad Nourdin

Morocco

Soliman Noureldin

UK

Nader Nouri-Majalan

Iran

Muhammed Mubarak Nuhari

Pakistan

Brian O'Donnell

Ireland

Olusola Oduntan

USA

Kayode Oduwole

Ireland

Yutaka Ogata

Japan

Masafumi Ohki

Japan

Ridvan Oksayan

Turkey
Francesco Oliva

Italy

Daniel Olmedo

Argentina

Kathleen Olson

USA

Sukru Sadik Oner

Turkey

Carlos Eduardo Ontiveros

Brazil

Anil Thomas Oommen

India

Ambrogio Orlando

Italy

Norberto Ortego-Centeno

Spain

Melissa Osborn

USA

Zdenko Ostojic

Bosnia and Herzegovina

Takanobu Otsuka

Japan

Toshinori Ozaki

Japan

Aynur Özge

Turkey

David Pace

Malta

Clifford Packer

USA

Ivan Padjen

Croatia

Dasja Pajkrt

Netherlands

Ajay Pal Singh

India

Aparna Palit

India

Jose Prisco Palma-Nicolas

Mexico

Periklis Panagopoulos

Greece

Vijay Panchanadikar

India
Padmakar Pandit

India

Amit Pandya

USA

Stavros Panidis

Greece

Maria Panopoulou

Greece

Vasileios Papadopoulos

Greece

Kostas Papagiannopoulos

UK

Theodossis S. Papavramidis

Greece

Basilios Papaziogas

Greece

Spyros Papiris

Greece

Dimitrios Paraskevopoulos

UK

Paolo Paredi

UK

Lawrence Parish

USA

Jeong-Yeol Park

South Korea

Jung Gil Park

South Korea

Jong Sung Park

South Korea

Martyn Parker

UK

Rocio Parody

Spain

Nigel John Parr

UK

Marco Pasini

Italy

Dave Paskar

Canada

Dritan Pasku

Greece

Gregorios Paspatis

Greece 
Salvatore Patanè

Italy

Anil Pathare

Oman

Anil Patki

Friedemann Paul

Germany

Catherine Pennington

UK

Nadia Peparini

Italy

Bruno Pereira

Brazil

Ajantha Perera

Sri Lanka

Nilanka Perera

Sri Lanka

Maria Asuncion Perez-Jacoiste Spain

Aleksandar Peric

Serbia

Christian-Dominik Peterlein

Germany

Adriano Piattelli

Italy

Gabriele Piffaretti

Italy

Promod Pillai

USA

Paolo Pillastrini

Italy

Trinitario Pina Murcia

Spain

Antonio Pinto

Italy

Chrysoula Pipili

Greece

Francesco Pisani

Italy

Mauro Pittiruti

Italy

Benjamin Piura

Israel
Domenico Plantone

Italy

Mike Polyzonis

Greece

\section{A. Ponnuswamy \\ UK \\ Vera Popovic \\ Serbia \\ Ramin Pourghorban \\ Iran \\ Harry Powell \\ UK \\ Juan Carlos Prados-Frutos \\ Spain}

Manousos-Georgios Pramateftakis

Greece

Sampath Chandra Prasad

India

Carlo Pratesi

Italy

Karl-Josef Prommersberger

Germany

Anna Punga

Sweden

Sohail Qayyum

USA

Aldo Quattrone

Italy

Ahmed Rabie

Egypt

Jasim Radhi

Canada

Annie Rajaratnam

India

Juan Fernando Ramon

Colombia

Serramito Ramon

Spain

Bruce Ramshaw

USA

Murali Rangaswami, Tanjore India

Piyush Ranjan

India
Anirudh Rao

UK

Amrith Rao

UK

Dimitrios Raptis

Germany

Serge Rasskazoff

USA

Barbara Rath

Germany

Chamara Ratnayake

Sri Lanka

Hilpi Rautelin

Sweden

Richter Razafindratsimandresy

Madagascar

Massimo Re

Italy

Krishna Reddy

UK

Nishitha Reddy

USA

Neil Reeves

UK

Roberto Ria

Italy

Benjamin Ricciardi

USA

Jonathan Riess

USA

Richard Rison

USA

Hisashi Ro

Japan

Simon Benedict Roberts

UK

Damith Rodrigo

Sri Lanka

Udo Rolle

Germany

Pol Maria Rommens

Germany

Avi Rosenberg

USA 


\begin{tabular}{|c|c|c|}
\hline $\begin{array}{l}\text { Andrew Rosenstengel } \\
\text { Australia }\end{array}$ & $\begin{array}{l}\text { Ami Schattner } \\
\text { Israel }\end{array}$ & $\begin{array}{l}\text { Samuel Shinjo } \\
\text { Brazil }\end{array}$ \\
\hline $\begin{array}{l}\text { Hai-Bin Ruan } \\
\text { USA }\end{array}$ & $\begin{array}{l}\text { Sam Schulman } \\
\text { Canada }\end{array}$ & $\begin{array}{l}\text { Manabu Shirotani } \\
\text { Japan }\end{array}$ \\
\hline $\begin{array}{l}\text { Vidyendaran Rudhran } \\
\text { India }\end{array}$ & $\begin{array}{l}\text { Cara Sedney } \\
\text { USA }\end{array}$ & $\begin{array}{l}\text { Jun Shoji } \\
\text { USA }\end{array}$ \\
\hline $\begin{array}{l}\text { Erin Rudzinski } \\
\text { USA }\end{array}$ & $\begin{array}{l}\text { Joern Seeger } \\
\text { Germany }\end{array}$ & $\begin{array}{l}\text { Rachel Shparberg } \\
\text { Australia }\end{array}$ \\
\hline $\begin{array}{l}\text { Wadia Rustom } \\
\text { India }\end{array}$ & $\begin{array}{l}\text { Stefan Seewald } \\
\text { USA }\end{array}$ & $\begin{array}{l}\text { Brian Shuch } \\
\text { USA }\end{array}$ \\
\hline $\begin{array}{l}\text { John Rutka } \\
\text { Canada }\end{array}$ & $\begin{array}{l}\text { Salih Selek } \\
\text { Turkey }\end{array}$ & $\begin{array}{l}\text { Aseem Shukla } \\
\text { USA }\end{array}$ \\
\hline $\begin{array}{l}\text { Aly Saber } \\
\text { Egypt }\end{array}$ & $\begin{array}{l}\text { Sixten Selleng } \\
\text { Germany }\end{array}$ & $\begin{array}{l}\text { Dhaval Shukla } \\
\text { India }\end{array}$ \\
\hline $\begin{array}{l}\text { Amitabh Saha } \\
\text { India }\end{array}$ & $\begin{array}{l}\text { Dhivakar Selvaraj } \\
\text { India }\end{array}$ & $\begin{array}{l}\text { Bobby Siddiqui } \\
\text { UK }\end{array}$ \\
\hline $\begin{array}{l}\text { Edith Said } \\
\text { Malta }\end{array}$ & $\begin{array}{l}\text { Seyedmojtaba Seyedmousavi } \\
\text { Netherlands }\end{array}$ & $\begin{array}{l}\text { Christos Sidiropoulos } \\
\text { USA }\end{array}$ \\
\hline $\begin{array}{l}\text { Mehmet Coskun Salman } \\
\text { Turkey }\end{array}$ & $\begin{array}{l}\text { Daniel Shaerf } \\
\text { UK }\end{array}$ & $\begin{array}{l}\text { Alicja Sieminska } \\
\text { Poland }\end{array}$ \\
\hline $\begin{array}{l}\text { Carmelo Salpietro } \\
\text { Italy }\end{array}$ & $\begin{array}{l}\text { Hetalkumar Shah } \\
\text { India }\end{array}$ & $\begin{array}{l}\text { Francesco Signorelli } \\
\text { France }\end{array}$ \\
\hline $\begin{array}{l}\text { Panagiotis Samaras } \\
\text { Switzerland }\end{array}$ & $\begin{array}{l}\text { Abdul-Rahman Shahein } \\
\text { Canada }\end{array}$ & $\begin{array}{l}\text { Anjana Silva } \\
\text { Sri Lanka }\end{array}$ \\
\hline $\begin{array}{l}\text { Dayananda Samarawickrama } \\
\text { UK }\end{array}$ & $\begin{array}{l}\text { Mahesh Shanmugam } \\
\text { India }\end{array}$ & $\begin{array}{l}\text { Francisco Javier Silvestre } \\
\text { Spain }\end{array}$ \\
\hline $\begin{array}{l}\text { Athanasios Saratzis } \\
\text { UK }\end{array}$ & $\begin{array}{l}\text { Sapna Sharan } \\
\text { Canada }\end{array}$ & $\begin{array}{l}\text { Charles Simone } \\
\text { USA }\end{array}$ \\
\hline $\begin{array}{l}\text { Aqiba Sarfraz } \\
\text { Pakistan }\end{array}$ & $\begin{array}{l}\text { Aanchal Sharma } \\
\text { India }\end{array}$ & $\begin{array}{l}\text { Jory Simpson } \\
\text { Canada }\end{array}$ \\
\hline $\begin{array}{l}\text { Maria Sarigianni } \\
\text { Greece }\end{array}$ & $\begin{array}{l}\text { Shilpa Sharma } \\
\text { India }\end{array}$ & $\begin{array}{l}\text { Chanchal Singh } \\
\text { India }\end{array}$ \\
\hline $\begin{array}{l}\text { Takeshi Sasaki } \\
\text { USA }\end{array}$ & $\begin{array}{l}\text { Rohit Sharma } \\
\text { India }\end{array}$ & $\begin{array}{l}\text { T. Shantikumar Singh } \\
\text { India }\end{array}$ \\
\hline $\begin{array}{l}\text { Kanji Sasaki } \\
\text { Japan }\end{array}$ & $\begin{array}{l}\text { Sameh Shehata } \\
\text { Egypt }\end{array}$ & $\begin{array}{l}\text { Nicholas Slater } \\
\text { Netherlands }\end{array}$ \\
\hline $\begin{array}{l}\text { Terence Savaridas } \\
\text { UK }\end{array}$ & $\begin{array}{l}\text { Sherif Shehata } \\
\text { Egypt }\end{array}$ & $\begin{array}{l}\text { Víctor Slavutsky } \\
\text { Spain }\end{array}$ \\
\hline $\begin{array}{l}\text { Sandro Sbordone } \\
\text { Italy }\end{array}$ & $\begin{array}{l}\text { Manjunath Shenoy } \\
\text { India }\end{array}$ & $\begin{array}{l}\text { Benjamin Smallheer } \\
\text { USA }\end{array}$ \\
\hline $\begin{array}{l}\text { Marius Scarlat } \\
\text { France }\end{array}$ & $\begin{array}{l}\text { Takahiko Shibahara } \\
\text { Japan }\end{array}$ & $\begin{array}{l}\text { Stuart Smith } \\
\text { UK }\end{array}$ \\
\hline $\begin{array}{l}\text { Bernhard Schaller } \\
\text { France }\end{array}$ & $\begin{array}{l}\text { Satoru Shikata } \\
\text { Japan }\end{array}$ & $\begin{array}{l}\text { Henry Smithson } \\
\text { Ireland }\end{array}$ \\
\hline
\end{tabular}


Ioannis Sokolakis

Greece

Doriette Soler

Malta

Sang Heon Song

South Korea

Lei Song

China

Guy Soo Hoo

USA

Shashank Sood

India

Toma Spiriev

Bulgaria

Giuseppe Spoto

Italy

Thambipillai Sri Paran

Ireland

Samer Srour

USA

Zdenek Stach

Czech Republic

Joerg Stein

Austria

Paschalis Steiropoulos

Greece

Anna Stepniewska

Italy

Hugo St-Hilaire

USA

Jacob Strahilevitz

Israel

Yoshiyuki Suehara

Japan

Koichiro Sugimura

Japan

Ruifang Sui

China

Sumadiono

Indonesia

Tucheng Sun

China

Rafal Surmacz

Poland
Alexey Surov

Germany

Maarten Taal

UK

Mariam Tajir

Morocco

Zennure Takci

Turkey

Tsuneo Takebayashi

Japan

Robert Takes

Netherlands

Nagio Takigawa

Japan

Anil Takvani

India

Winston Tan

USA

Tadashi Tanaka

Japan

Nil Tekin

Turkey

Nina Tello-Winniczuk

Mexico

H. Thomas Temple

USA

Abdulkadir Tepeler

Turkey

Hardik Thakker

India

Jagdeep Thakur

India

Rokshana Thanadar

USA

Loukas Thanos

Greece

Calvin Thigpen

USA

C.R. Thomas

USA

Dick Tibboel

Netherlands

Holger Till

Austria
Elwina Timehin

UK

Hiroyuki Tokue

Japan

Dario Tomasini

Italy

Benedetta Tomberli

Italy

Vincenzo Tombolini

Italy

Christopher Tormey

USA

Konstantinos Toulis

Greece

D.E. Tran

Canada

Christine Tranchant

France

Jonel Trebicka

Germany

Gianluca Trifiro

Italy

Chen-Chi Tsai

Taiwan

Stella Tsai

Taiwan

Iraklis Tsangaris

Greece

Ioannis Tsarouhas

Greece

Dimitrios Tsetis

Greece

Parmenion Tsitsopoulos

Sweden

Norihiko Tsuchiya

Japan

Mehmet Turgut

Turkey

Stefano Uccella

Italy

Kenzo Uchida

Japan

Akin Ugras

Turkey 


\author{
Burak Veli Ulger \\ Turkey \\ Claudio Ungari \\ Italy
}

Gabor Ungvari

Australia

Agahan Unlu

Turkey

Rashmi Upadhyay

India

Ofir Uri

Israel

Nicolas Useche

Colombia

Jiri Valenta

Czech Republic

Virginia Van Duyne

USA

Vincent Vander Poorten

Belgium

Ivor Vanhegan

UK

Prasad Vannemreddy

India

Nikolaos Vassos

Germany

P. Veena

India

Giovanni Vennarecci

Italy

Jose Ventura

USA

Stefano Veraldi

Italy

David Verity

UK

Paolo Verze

USA

Nereo Vettoretto

Italy

Kenneth Vick

USA

Goran Videnovic

Serbia
Ana Paula Vivas

Brazil

Theodosia Vogiatzaki

Greece

Jesse Waggoner

USA

Santosh Waigankar

India

Heather Wakelee

USA

Daniel Walsh

UK

Michael Walton

UK

Wei-Jie Wang

Taiwan

Hong Wang

China

Yuhui Wang

China

Alison Ward

Canada

David Warrell

UK

Atsuya Watanabe

Japan

Sama Weerapperuma

Sri Lanka

Öghdf Wefwfr

Turkey

Eva Wegner

Australia

Joachim Weis

Germany

Mandika Wijeyaratne

Sri Lanka

James Wilson

USA

James Winearls

Australia

Guido Woeste

Germany

Ronit Wollstein

USA
Samuel Ys Wong

Hong Kong

Seung Hoon Woo

South Korea

Sandy Wright

Canada

Tsung-Tien Wu

Taiwan

Shoji Yabuki

Japan

Naveen Yadav

India

Yoshitaka Yamaguchi

Japan

Akira Yamasaki

Japan

Hiroshi Yamazaki

Japan

Yoko Yanagisawa

UK

Yukihiro Yano

Japan

Alpaslan Yavuz

Turkey

Dali Yin

USA

Hiroshi Yoshida

Japan

Cheol Woong Yu

South Korea

Canan Yücesan

Turkey

Hideo Yuki

Japan

Zacharias Zachariah

Switzerland

Yehuda Zadik

Israel

Ahmed M. Zaki

Egypt

Apostolos Zatagias

Greece

Nick Zavras

Greece 
Itai Zeevi

Israel

Philippe Zerbib

France

Haitao Zhao

China

Chunlong Zhong

China

Jun Zhong

China
Jasna Zidverc-Trajkovic

Serbia

Esther Zipperer

Germany

Richard Zoraster

USA

Konstantinos Zorbas

Greece

Giovanna Zorzi

Italy
Vasiliki Zouvelou

Greece

Alauldeen Zubair

Iraq

Ali Ersin Zumrutbas

Turkey 